\title{
Case Based Methodology: A Method to Enhance the Learning of Physiological Basis of Cardiovascular and Respiratory System to Undergraduate Medical Students
}

\author{
Puja Dulloo $^{1, *}$, Nirmal A. Pathare ${ }^{2}$ \\ ${ }^{1}$ Department of Physiology, Oman Medical College, Bausher, Muscat, Sultanate of Oman \\ ${ }^{2}$ Department of Natural Science, Oman Medical College, Bausher, Muscat, Sultanate of Oman \\ *Corresponding author: pujadulloo2012@gmail.com
}

Received November 09, 2013; Revised November 17, 2013; Accepted November 25, 2013

\begin{abstract}
Introduction: Case based teaching methodology is a discrete approach to interactive group- based learning. Its implementation along with didactic teaching schedule has an added advantage by evoking interest in students by placing them in a dilemma taken from a real- life example and motivates them for self-directed learning. The aim of the present exercise was to introduce the students to an alternative method of learning compared with the traditional one used, to enhance self- directed learning and correlate the theoretical contents with its applied aspect. Method: A study was done in spring semester with $2^{\text {nd }}$ year medical students at OMC, Muscat, Oman. Didactic lecture followed by case study after few days on ECG topic from CVS was taught. Four random groups were made within the class having a student leader for coordination. Pre-test was conducted before a clinical case scenario with applied MCQ's were discussed week after the topic taught which was followed by post- test. Another clinical case dealing with respiratory system was discussed within the semester in a similar fashion. Feedback survey questionnaire with a response of 5 points Likert's scale was filled by participant after completion of the course. Focus group discussion was conducted among randomly selected 15 students to have in-depth feedback about methodology. Result: Out of 95, 91 students qualified the inclusion criteria. Statistically significant increase in the academic score were obtained on the topics taught by CBM $(\mathrm{p}<0.005)$ from pre and post test. Descriptive statistical analysis for 28 questions showed positive response by $92.3 \%$ students for improvement in their knowledge related to human physiology while $83.5 \%$ accepted that the methodology helped them to relate topic with the clinical situation. While $75.9 \%$ of students accepted that CBM encouraged them to use various resources material. In focus group discussion students commented that methodology helped them to correlate theoretical information with its clinical application and they started reading more books and gathers information from other resources. Conclusion: Case based teaching provides opportunity for students to use different resource materials and clarify the concepts, other than have better interaction with the instructor.
\end{abstract}

Keywords: case based methodology, student centered learning, self-directed learning approach, physiology

Cite This Article: Puja Dulloo, and Nirmal A. Pathare, "American Journal of Educational Research." American Journal of Educational Research 1, no. 10 (2013): 425-429. doi: 10.12691/education-1-10-3.

\section{Back Ground}

The way students approach their learning plays an important role in determining the outcome of any educational endeavor. It determines the quality and quantity of education and the learning approach of the students. The quality and approach is influenced by a number of factors, such as teaching characteristics, departmental characteristics and assessment methods [1]. Researchers in medical education have identified the three basic learning approaches adopted by medical students. They are surface, deep and strategic [2].

The undergraduate medical students have many challenges and hurdles to face all along their way. The deep approach is the most appropriate and desirable way of learning that is closely linked to the intellectual thinking we would wish to see in all medical students. These are the means of lifelong learning [3]. In order to help the students to be better learner' the medical knowledge should be learned in context of a clinical case that actually would better comprehend and be easily applicable as deep approach of learning.

Problem Based Learning (PBL) is an educational approach that is being implemented differently in different medical schools. PBL was first introduced by the Mc Masters University Medical School in Hamilton, Ontario, Canada in late 1960's by Howard Barrows and his colleagues as an educational format centered around the discussion and learning that emanates from a clinical based problem [4]. The case method, a discrete approach to interactive group- based learning, was already introduced a century ago within economics and the faculty 
of law[5].The aim of the case i.e., to gain familiarity with knowledge already acquired by teacher- directed learning is simpler and easier to realize where in PBL learning is self generation of knowledge under the supervision of a process facilitator.

Due to a more teacher- dependent approach to learning, the case method is less susceptible to group dysfunction which is well recognized problem inherent in the PBL group. Other than this limitation in PBL, students jump directly to diagnostic conclusions and go through medicine textbooks to find out the treatment protocol for the disorder rather than understanding the basis of disease and its comparison with the normal physiological basis [6]. In PBL, sources for knowledge are wide ranged, starting from lectures and library and going up to web and classification of text and learning factual knowledge by self study. While in case based learning source material is restricted by the extent of factual knowledge and classification of the text is to better extend without utilization of excess time. In spite of having various advantages in CBT compared to PBL there are a few limitations to this method. The case should have a real background, irritates or triggers willingness to discuss and concomitantly fulfils scheduled learning objectives [5].The case method is less resource consuming, primarily because it can be practiced in groups several times larger than those of the PBL tutorial [7]. This concept made us think to add some of the clinical cases in physiology and microbiology in order to make the concept much clearer clinically.

The medical schools in Oman do have PBL in their curriculum but adding Case Based learning during didactic teaching schedule had an added advantage by evoking interest in students by placing them in a dilemma taken from a real- life example. The students have opportunities for familiarization and deepening of knowledge already acquired through lectures and other sources. The students having any knowledge gaps regarding the taught topics can open to the teachers in case based learning. Thus there will be improvement in learning concepts in the students and deep structural learning will develop within them.

Specific criteria to take a clinical case for discussion to basic science medical students include the following features:

- It should not be too complicated or special

- There is a need for capable teacher for chairing case sessions; which includes ability to listen, catch signals in the class room and make students talk to each other.

\section{Objectives}

- Compare the performance of students for the topics covered during didactic lecture and after case based teaching combined with the didactic lecture.

- Study the effect of CBT for self directed learning.

\section{Methodology}

In spring semester 2012, a quantitative study was done with $2^{\text {nd }}$ year medical students at Oman medical college,
Muscat, Oman. 95 students participated out of which only 91 qualified the inclusion criteria for interpretation of the results. The students were in the age group of 18-20 years and were of varied academic background i.e., having basic education in English medium and Arabic medium at school education level.

The teaching module consisted of mainly didactic lectures, tutorials and practical classes based on the topics related to Cardio-vascular and Respiratory systems. Four lecture classes and two tutorial classes per week were scheduled other than two office hours for the students.

The Case based problem solving learning module was introduced during a separate class scheduled for the same reason for the entire duration.

The students were taught the ECG topic from CVS via didactic lecture which was followed by a case study based on the same topic after a few days.

The class was divided into four groups randomly and one leader was chosen from the group for proper coordination.

The students were provided a week's time to discuss the case and work over the questions provided along with the case with a physiological reason for the answer. All the four groups came together and filled a pre post for the topic. Then the case was discussed openly among the groups and questions were solved and further concepts were clarified by the faculty members. Then a post test was given to the students.

Care was taken regarding the following aspects:

- Pre- post test questions were briefly and correctly formulated to match the level of the students with previously acquired knowledge covered in the didactic lecture classes.

- Cases/ problems based questions were discussed properly and systematically.

- Each member of the group had a chance to participate over and above the leader

- The students did not attempt to jump to conclusions and find a diagnosis of the disease.

- Some students deliberately avoided the tutorial classes and participation either due to language barrier or other varied reasons. The faculty tried to communicate with them by different moderator sessions so that they could easily participate in the case discussion and asked the leader to make them work over the case and participate fully.

Sufficient time was given to discuss the case within the group by referring to books and internet sites.

Similarly other clinical case was discussed related to respiratory system topic within the semester.

Throughout the spring semester this mode of teaching/ learning process continued in parallel along with didactic classes. At the end of the term, the students were given a questionnaire to put forward their opinion regarding the usefulness of the techniques.

The Questionnaire had 28 questions with a response of 5 points Likert's scale.

(Annexure I) It was framed in such a way that it yielded information regarding the following defined objectives:

- Has CBT improved the knowledge about that topic?

- Was integration appropriate to clarify the relationship of theoretical knowledge to the clinical practical aspect? 
- Where the skills appropriate to develop an analytical and critical thinking within themselves to solve challenging clinical cases?

- Did the clinical cases strengthen the link between the theory and practice of medicine so that the students could see how the questions in the clinical history were related to the basic understanding of the normal structure and functions so that its interpretation became easier?

- Whether there had been a valuable exchange of ideas in small groups?

- Did the methodology encourage them to do self study and improve their resources usage?
Focus group discussion was conducted among 15 students for duration of 1hour and 45 minutes before filling Survey questionnaire. Notes for the focus group were taken by two faculty members and one senior faculty member facilitated the discussion for smooth conduction. (Annexure II \& III)

\section{Results}

Pre and post test on CVS and respiratory system for specific topics was conducted.

The paired sample test for both showed a statistical significance $(\mathrm{p}<0.005)$ Table 1 and Table 2 and Figure 1.

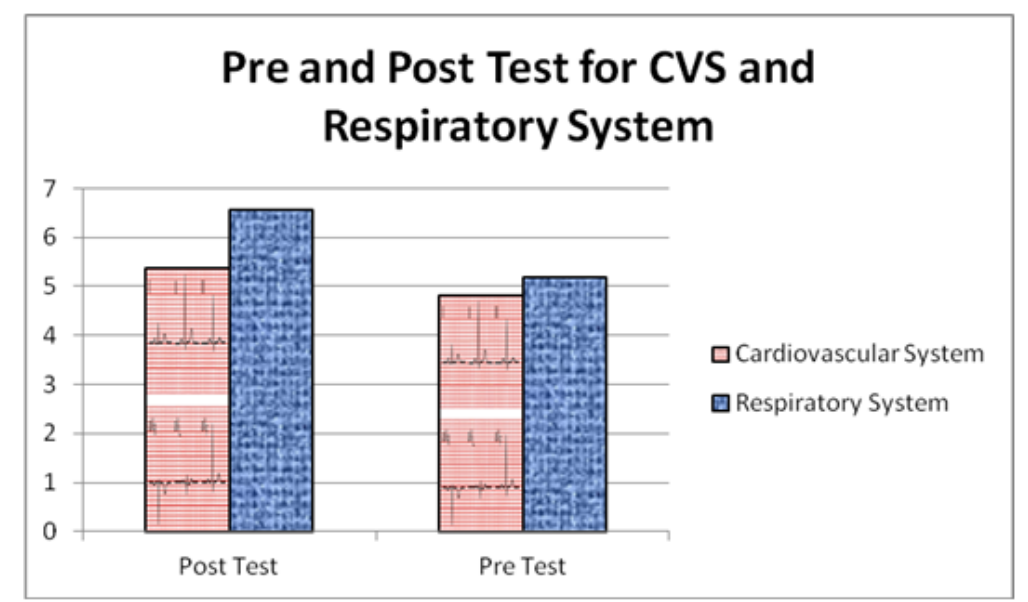

Figure 1.

Table 1. Paired Samples Test

\begin{tabular}{|c|c|c|c|c|c|c|c|c|c|}
\hline & \multicolumn{5}{|c|}{ Paired Differences } & \multirow{3}{*}{$\mathrm{t}$} & \multirow{3}{*}{ df } & \multirow{3}{*}{$\begin{array}{l}\text { Sig. (2- } \\
\text { tailed) }\end{array}$} \\
\hline & & \multirow{2}{*}{ Mean } & \multirow{2}{*}{$\begin{array}{c}\text { Std. } \\
\text { Deviation }\end{array}$} & \multirow{2}{*}{$\begin{array}{l}\text { Std. Error } \\
\text { Mean }\end{array}$} & \multicolumn{2}{|c|}{$\begin{array}{l}\text { 95\% Confidence Interval of the } \\
\text { Difference }\end{array}$} & & & \\
\hline & & & & & Lower & Upper & & & \\
\hline $\begin{array}{c}\text { Pair } \\
1\end{array}$ & $\begin{array}{c}\text { Post test CVS - Pre test } \\
\text { CVS }\end{array}$ & 0.542 & 1.517 & 0.166 & 0.211 & 0.873 & 3.257 & 82 & .002 \\
\hline
\end{tabular}

Table 2. Paired Samples Test

\begin{tabular}{|c|c|c|c|c|c|c|c|c|c|}
\hline & \multicolumn{5}{|c|}{ Paired Differences } & \multirow{3}{*}{$\mathrm{t}$} & \multirow{3}{*}{ df } & \multirow{3}{*}{$\begin{array}{l}\text { Sig. (2- } \\
\text { tailed) }\end{array}$} \\
\hline & & \multirow{2}{*}{ Mean } & \multirow{2}{*}{$\begin{array}{c}\text { Std. } \\
\text { Deviation }\end{array}$} & \multirow{2}{*}{$\begin{array}{l}\text { Std. Error } \\
\text { Mean }\end{array}$} & \multicolumn{2}{|c|}{$\begin{array}{l}\text { 95\% Confidence Interval of the } \\
\text { Difference }\end{array}$} & & & \\
\hline & & & & & Lower & Upper & & & \\
\hline $\begin{array}{c}\text { Pair } \\
1\end{array}$ & $\begin{array}{l}\text { Post test RS - Pre test } \\
\text { RS }\end{array}$ & 1.375 & 1.118 & 0.125 & 1.126 & 1.624 & 11.000 & 79 & .000 \\
\hline
\end{tabular}

The descriptive statistical analysis for 28 questions done by using SPSS-19 which showed that maximum mean for all the questions ranged from 2.43 to 4.52 . The maximum rank was for question 5 stating that clinical

cases were in context of the system taught currently followed by question 6 stating that CBT methodology helped them to improve knowledge of Human physiology. (Table 3 and Figure 2).

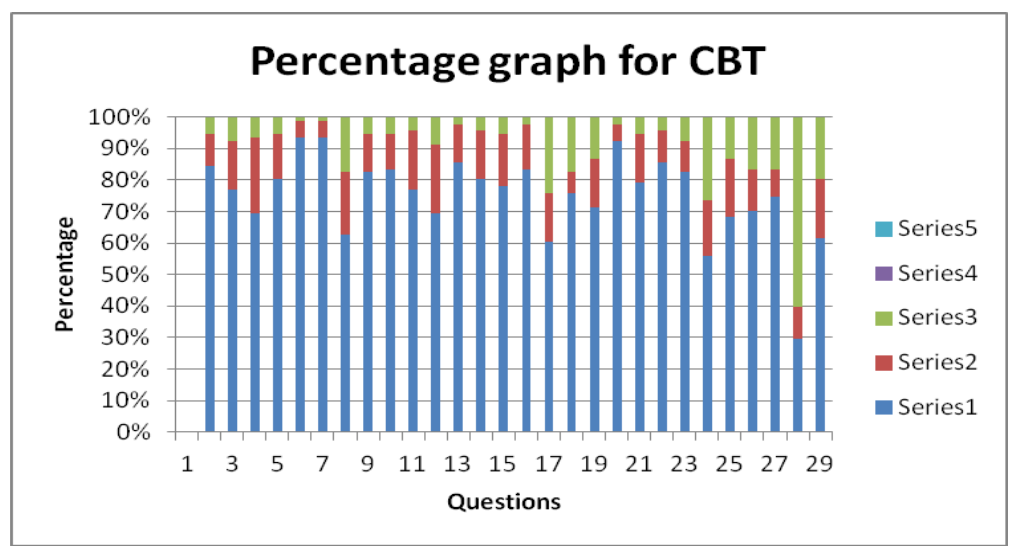

Figure 2. 
Table 3.

\begin{tabular}{|c|c|c|c|c|}
\hline \\
\hline Q.No. & QUESTION & MEAN & SD & $\begin{array}{c}\text { Percentage of } \\
\text { positive response* }\end{array}$ \\
\hline 1 & This mode of teaching helps to achieve the unit's general objectives & 3.98 & 0.84 & 84.62 \\
\hline 2 & The resource sessions help to understand difficult/ complex topics & 3.90 & 0.96 & 76.92 \\
\hline 3 & $\begin{array}{c}\text { The resource sessions allow better understanding of the co-ordinate action of } \\
\text { several systems in the homeostatic response }\end{array}$ & 3.84 & 0.84 & 69.23 \\
\hline 4 & The course increased my interest in the topic taught & 4.00 & 0.83 & 80.22 \\
\hline 5 & $\begin{array}{l}\text { The clinical cases given in physiology were in the context of the system currently } \\
\text { studied }\end{array}$ & 4.52 & 0.65 & 93.41 \\
\hline 6 & This methodology has helped to improve my knowledge of human physiology & 4.30 & 0.69 & 93.41 \\
\hline 7 & The time duration provided for the students to discuss the questions was adequate & 3.65 & 1.11 & 62.64 \\
\hline 8 & Questions were directly related to the case discussed & 4.09 & 0.89 & 82.42 \\
\hline 9 & The course enabled me to relate with clinical situations & 4.05 & 0.94 & 83.52 \\
\hline 10 & The course method helped me integrate different aspects of the topic & 3.93 & 0.94 & 76.92 \\
\hline 11 & The sessions enhanced interaction between instructor and student & 3.85 & 1.12 & 69.23 \\
\hline 12 & $\begin{array}{c}\text { The learning acquired through these sessions allowed me to appreciate human } \\
\text { body function in an integrated manner }\end{array}$ & 4.14 & 1.05 & 85.71 \\
\hline 13 & This method has facilitated collaborative learning among students & 3.98 & 1.16 & 80.22 \\
\hline 14 & The course method succeeded in promoting problem solving skills & 4.04 & 1.30 & 78.02 \\
\hline 15 & $\begin{array}{l}\text { The method enabled me to apply the knowledge of basic Science for clinical } \\
\text { reasoning }\end{array}$ & 4.11 & 1.36 & 83.52 \\
\hline 16 & Usage of resources increased while studying cases (Books) & 3.65 & 1.79 & 60.44 \\
\hline 17 & Cases study encouraged me to use more of internet resources & 3.98 & 1.72 & 75.82 \\
\hline 18 & $\begin{array}{l}\text { The availability of additional resource material regarding the course helped in } \\
\text { self-study }\end{array}$ & 3.9891 & 1.776 & 71.43 \\
\hline 19 & $\begin{array}{l}\text { The cases studies posed challenging questions that helped me develop analytical } \\
\text { and critical thinking }\end{array}$ & 4.44 & 1.67 & 92.31 \\
\hline 20 & The laboratory exercises co-ordinate with the theory being taught & 4.24 & 1.88 & 79.12 \\
\hline 21 & $\begin{array}{l}\text { The co-ordination of theory and lab during this course helped me understand the } \\
\text { topic in a better way }\end{array}$ & 4.40 & 1.94 & 85.71 \\
\hline 22 & $\begin{array}{c}\text { The co-ordination of theory and lab during this course helped me improve my } \\
\text { reasoning skills }\end{array}$ & 4.2174 & 2.048 & 82.42 \\
\hline 23 & There are some gaps in knowledge after completing my study of this course & 3.58 & 2.33 & 56.04 \\
\hline 24 & Overall, I am satisfied with the course methodology used during the session & 3.86 & 2.32 & 68.13 \\
\hline 25 & I think there should be more cases included per semester & 4.01 & 2.51 & 70.33 \\
\hline 26 & I think more time should be devoted for conduct of these sessions & 4.17 & 2.55 & 74.73 \\
\hline 27 & Case study related questions should be added to graded exams/ quizzes & 2.70 & 2.91 & 29.67 \\
\hline 28 & I consider CBT being more informative and motivating compared to PBL sessions & 3.94 & 2.81 & 61.54 \\
\hline & *Agreed and Strongly agreed & & & \\
\hline
\end{tabular}

Table 4.

\section{KEY QUESTION 1: How was Case based teaching beneficial to you?}

Comments:

- We could correlate the theoretical information with that of its application in near future.

- Few said that it created confusion at some points which cleared off after faculty discussion.

- Time duration provided for preparing the case was less initially but by repeated exposure we got tuned to it.

- Understanding of reading medical reports improved.

- Sometime the extent of information could not be levelled.

- This improved our knowledge (previous with present and then future).

- We started reading more books from the library and tried to gather information from net.

- We had a feeling that we are going to be doctors and started thinking like that.

- We had a better understanding and co-ordination with the instructor.

- Some cases did help in problem solving skill.

- Now I know how to study in a medical school

Table 5.

\section{KEY QUESTION 2: How can it be improved?}

\section{Comments:}

- Big group had less participation; when instructor divided us into 4 groups it worked in a better way

- Small groups should be for discussing the case so that everyone could get a chance to participate.

- In formative assessments less wattage should be given to critical thinking questions.

- More short cases should be given at the end of every class.

- Grades should be given as per discussion in the cases 
Table 6.

KEY QUESTION 3: Anything specific?

Comments:

- Few liked PBL and few said CBT but on an average CBT was considered preferable mode of teaching.

- Majority concluded that case study related questions should be added to our formative as well as summative exams

Focus group discussion was done among 15 students with 3 keys question and their comments are stated in Table 4, Table 5 and Table 6.

\section{Discussion}

There has always been a question whether Problem based tutorial or Case based teaching as a teaching module could be conducted in basic science courses and many universities have shown that it is possible.

The case studies have been proposed to promote active learning [8].

The present study used a case oriented format to promote inquiry driven learning in the second year medical undergraduate students.

61.6\% of students considered CBT more informative and motivating compared to PBL sessions and improvement in student comprehension of the subject material [9].

$74.8 \%$ of students thought that more time should be devoted for conducting CBT sessions and 70.3\% students thought that more cases should be included per semester.

$92.3 \%$ of students accepted that cases taught posed challenging questions that helped them develop analytical and critical thinking which was more than other studies $[10,11]$.

Learning needs to be an active constructive mental activity which makes one understand a particular area of knowledge. $75.9 \%$ of students believed that this methodology of teaching encouraged them to use internet resources. $60.5 \%$ of students accepted that the usage of book resources increased while studying cases thus giving them ample motivation to do self directed learning. It boosted their learning skills.

Case based teaching methodology served the primary objective and allowed students to handle it analytically and relate the course content with a clinical situation thus have active learning which was agreed by $83.5 \%$ of students [12,13].

This method of teaching was an initial attempt to familiarize the students with newer alternative methods of learning other than PBL to encourage them to work as a part of team within time constraint. [14] 69.2\% of students accepted that during the session, interaction between the instructor and student as well as amongst the students was enhanced.

$83 \%$ students of the batch passed the semester with more than $70 \%$ marks.

\section{Conclusions}

The case based teaching is a learner- centered teaching methodology where the students address problems as a group. Majority of the evidences outlined in the literature review also revealed that as a whole CBL is enjoyed by both students and the instructors.

\section{Acknowledgements}

I acknowledge the guidance and support provided by the Dean and senior as well as junior faculty members of OMC. I specifically would like to thanks students for their continuous engagement with this research. I would like to thank faculty of Foundation of Advancement of International Medical Education and Research for guiding and supporting me.

\section{References}

[1] NewbleDI, EntwistleNJ. "Learning styles and approaches: implications for medical education”. Med Educ 20;162-175: 1986.

[2] AbrahamRR, VinodP, KamathMG. "Learning approaches of undergraduate medical students to physiology in a non-PBL and partially PBL- oriented curriculum”. Adv Physiol Educ 32;35-37: 2008.

[3] MartonF, HounsellD, EntwistleNJ. "The experience of learning: Implications" Teaching and Learning in higher education Edinburgh: Scottish Academic 1997.

[4] Ghosh S. "Combination of didactic lectures and case- oriented problem- solving tutorials towards better learning: perceptions of students from a conventional medical curriculum”. Adv Physiol Educ 31;193-197: 2007.

[5] Barnes LB, ChristensenCR, HansenAB. "Teaching and the Case methods”. Boston, MA, Harvard Business school Press1994.

[6] William B. "Case based learning- a review of literature is there scope for the educational paradigm in pre-hospital education?" Emerg Med J 22; 577-581:2005.

[7] TarnvikA. "Revival of case methods: a way to retain studentcentered learning in a post PBL era”. Med Teacher 29;e32e36:2007.

[8] MayersC, JonesTB. "Case studies In: Promoting active learning: Strategies for the college classroom”. San Franciso, CA: JosseyBass; 103-119: 1993.

[9] CliffWH, AnnW. “ Directed Case study methods for teaching Human Anatomy and Physiology" Adv Phy Edu 15(1); S19S28:1996.

[10] CirajAM, VinodP, RamnarayanK. "Enhancing active learning in microbiology through case based learning: Experiences from an Indian medical school”. Indian J of Pathology and Microbiology; 53;729-733: 2010.

[11] RichardsP, InglehartMR. “An Interdisciplinary approach to case based teaching: Does it create patient centered and culturally sensitive providers?” J of Dental Edu 70(3); 284-291: 2006.

[12] SchapiroR, BeckerS, FoertschJA et al. "Integrative Cases for Preclinical Medical Students Connecting Clinical, Basic Science, and Public Health Approaches.” Am J Prev Med 41(4S3);S187S192: 2011.

[13] ChanWP, HsuCY, Hong CY. "Innovative "Case-Based Integrated Teaching” in an Undergraduate Medical Curriculum: Development and Teachers’ and Students’ Responses.” Ann Acad Med Singapore 37;952-6:2008.

[14] SrinivasanM, WilkesM et.al. "Comparing Problem based learning with Case based learning: Effects of a major curricular shift at two institutions” Academic Medicine 82(1); 74-82:2007. 